University of Nebraska - Lincoln

DigitalCommons@University of Nebraska - Lincoln

Faculty Publications from Nebraska Center for Materials and Nanoscience

Materials and Nanoscience, Nebraska Center for (NCMN)

$3-2010$

\title{
PCED2.0-A computer program for the simulation of polycrystalline electron diffraction pattern
}

\author{
Xingzhong Li \\ University of Nebraska - Lincoln, xli2@unl.edu
}

Follow this and additional works at: https://digitalcommons.unl.edu/cmrafacpub

Part of the Nanoscience and Nanotechnology Commons

$\mathrm{Li}$, Xingzhong, "PCED2.0-A computer program for the simulation of polycrystalline electron diffraction pattern" (2010). Faculty Publications from Nebraska Center for Materials and Nanoscience. 108.

https://digitalcommons.unl.edu/cmrafacpub/108

This Article is brought to you for free and open access by the Materials and Nanoscience, Nebraska Center for (NCMN) at DigitalCommons@University of Nebraska - Lincoln. It has been accepted for inclusion in Faculty Publications from Nebraska Center for Materials and Nanoscience by an authorized administrator of DigitalCommons@University of Nebraska - Lincoln. 


\title{
PCED2.0 - A computer program for the simulation of polycrystalline electron diffraction pattern
}

\author{
X. Z. Li \\ Nebraska Center for Materials and Nanoscience, University of Nebraska-Lincoln, \\ WSEC N104, Lincoln, NE 68588-0650, USA; email xli2@unl.edu
}

\begin{abstract}
A computer program for the simulation of polycrystalline electron diffraction patterns is described. PCED2.0, an upgraded version of the previous JECP/PCED, can be used as a teaching aid and research tool for phase identification, microstructure texture analysis, and phase fraction determination. In addition to kinematical theory for diffraction intensity calculation of polycrystalline samples, Blackman two-beam dynamical correction is included. March model is used for out-of-plane and in-plane texture simulation. A pseudo-Voigt function is used for the peak profile fitting of diffraction rings. User-friendly interface is improved in the handling of experimental diffraction data and the flexibility of indexing. Application of the program for the analysis of FePt thin films is given as an example.
\end{abstract}

Keywords: electron diffraction, polycrystalline phase, computer software

\section{Introduction}

For polycrystalline TEM specimens, including fine powders and particles, electron diffraction ring patterns are extensively used for phase identification, in which the diffraction pattern of a known phase acts as a fingerprint [1]. A computer program, JECP/PCED [2], was designed to provide a fast and accurate method for the task. The need for quantitative or semi-quantitative analysis of polycrystalline samples (powders and particles) requires the extension of the functions of the previous program. An upgraded version of the program, PCED2.0, has been developed to meet the requirement for phase identification, semi-quantitative analysis of structures and phases, and texture analysis and phase fraction determination.

Nowadays it is common to obtain full dynamic range of electron diffraction ring patterns digitally with the help of advanced techniques, such as image plates [3] and scientific grade charge-coupled device cameras [4]. Computer programs have been developed for processing polycrystalline electron diffraction patterns (e.g. $[5,6])$ in order to achieve better angular resolution, quantified intensities, and reproducible identification of discontinuous and/or faint rings. The extraction of integrated intensities from electron diffraction ring patterns makes it possible for quantitative (or semi-quantitative) analysis of the samples. The framework of such a development was discussed recently $[7,8]$ and computer programs were developed $[8,9]$.

Quantitative analysis of polycrystalline diffraction patterns consists of two basic steps: (i) digital processing of the experimental pattern to extract the quantitative data, and (ii) advanced simulation to interpret the data for phase analysis and/or structure determination and refinement. The PCED2.0 is developed for advanced simulation of polycrystalline electron diffraction patterns. New features in the PCED2.0 include: (i) Blackman two-beam dynamical correction of polycrystalline electron diffraction [10], (ii) March model for out-of-plane and in-plane texture [11], (iii) pseudo-Voigt peak profile fitting of diffraction rings, and (iv) the improved user-friendly interface in the handling of experimental diffraction data and the flexibility of indexing.

In the present paper, the concepts, models, and theories for developing the computer program PCED2.0 are briefly reviewed, and then the design and features of the program are described. Finally, the program is applied in the analysis of the diffraction patterns of FePt thin films as examples. A fully functional demonstration version of the program and a user's manual are available on the author's website: $\underline{\text { http:// }}$ www.unl.edu/CMRAcfem/XZLI/programs.htm. Readers who are interested in the full version of the program should contact the author.

\section{Background}

The kinematical theory of electron diffraction was implemented in the JECP/PCED and the PCED2.0. The Blackman formulae were included in the PCED2.0 to account for dynamical effects. The electron atomic scattering factor can be derived from X-ray atomic scattering factor from the Mott-Bethe relationship [12] or directly obtained from parameterized table of electron atomic scattering factor $[13,14]$. The second method was used in the PCED2.0. 
Following the electron diffraction geometry in the kinematical theory, the radius of the diffraction ring, $R$, can be derived from the reciprocal lattice vector $g(h k l)$ in second-order approximation as $[15,16]$

$$
R=L\left(\frac{g}{K}\right)\left(1+\frac{3}{8} \frac{g^{2}}{K^{2}}\right)
$$

where $L$ is the camera length, $g=|g(h k l)|$ the magnitude of reciprocal lattice vector, $K=|K|$ where $K$ is the wave vector of the incident electron beam.

\subsection{Blackman formulae for the electron diffraction intensity of polycrystalline phases}

Blackman [10] derived formulae for polycrystalline electron diffraction intensities based on Bloch wave dynamical theory using a two-beam approximation. The formulae were proved to be successful in later experimental work and extended to the structural analysis of polycrystalline phases [17]. The validation and limitation of the formulae were discussed by Cowley and Kuwabara [18]. Readers should refer to the original papers for detailed derivations. Some important equations are presented here using the conventions in the book by Spence and Zuo [12].

The dynamical structure factor $\left(U_{g}\right)$ is given as

$$
U_{g}=\frac{2 m e V_{g}}{h^{2}}
$$

where $m$ is the electron mass, $e$ is the electron charge, $h$ is the Planck's constant, and $V_{g}$ is the Fourier coefficient of the crystal potential.

The wave vector in vacuum $\left(K_{0}\right)$ is given as

$$
K_{0}^{2}=\frac{2 m e E}{h^{2}}
$$

where $E$ is the acceleration voltage of the electron microscope.

The wave vector inside the crystal $(K)$ is corrected using the mean inner potential $\left(U_{0}\right)$,

$$
K^{2}=K_{0}^{2}+U_{0}
$$

The diffraction intensity formula can be given in an integral form considering there are angle distributions between the incident beam and the zone axis of the polycrystalline grains

$$
I_{g}^{\text {integral }}=I_{0} \frac{U_{g}}{g K^{2}} \int_{0}^{\mathrm{A}} J_{0}(2 x) d x
$$

where $I_{0}$ is the intensity of an incident beam, $U_{g}$ the dynamical structure factor, $g$ the magnitude of a reciprocall lattice vector, $K$ the value of the wave vector, and $J_{0}(x)$ the zero-order Bessel function and

$$
A=\frac{\pi U_{g} t}{K_{n}}
$$

where $t$ is the crystal thickness, $K^{2}=K_{n}^{2}+K_{t}^{2}, K_{n}$ is the normal component, and $K_{t}$ is the component in the zero-order Laue zone.

The final intensity can be derived as an averaged integral intensity over $K_{n}$ considering the angle between the incident beam and the surface normal is in a range from $-(\pi / 4)$ to $\pi / 4$.

By considering $K_{n} \approx K$ as a further approximation, the intensity relation of the integrated two-beam dynamical treatment and the kinematical theory can be derived as [17]

$$
\frac{I_{g}^{\text {integral }}}{I_{g}^{\text {kine }}}=\frac{1}{\mathrm{~A}} \int_{0}^{\mathrm{A}} J_{0}(2 x) d x
$$

The implementation of the formulae for the averaged integral intensity over $K_{n}$ will cost more time in calculation but it gives more accurate results. These formulae were adopted in the PCEC2.0 since it uses the Blackman theory only at the final calculation. Further approximation as Equation (7) was used in the other programs which were implemented through the Blackman theory in the refinements routine for intensive calculation.

As expected, the calculation with the dynamical theory will give the same results as those obtained with the kinematical theory if the thickness of sample is thin enough. However, Cowley and Kuwabara [18] pointed out that the kinematical theory represented the correct limiting case for $t \lambda \rightarrow 0$, where $t$ is the crystal thickness and $\lambda$ is the wavelength of an incident electron beam, but not for $t \rightarrow 0$. This indicates that the Blackman formulae have their limitation although they are more accurate than the kinematical theory of diffraction intensities.

\subsection{Pseudo-Voigt function for peak profile fitting}

Although the Voigt $(V)$ function, as a convolution of Gaussian $(G)$ and Lorentzian $(L)$ functions, is considered the most suitable function to describe the peak profile of the diffraction rings, it is rather complex. The simpler pseudo-Voigt $(p V)$ function is usually sufficient to fit the peak profile. The pseudo-Voigt function is a linear combination of Gaussian and Lorentzian functions, represented by peak height $(h)$ and full

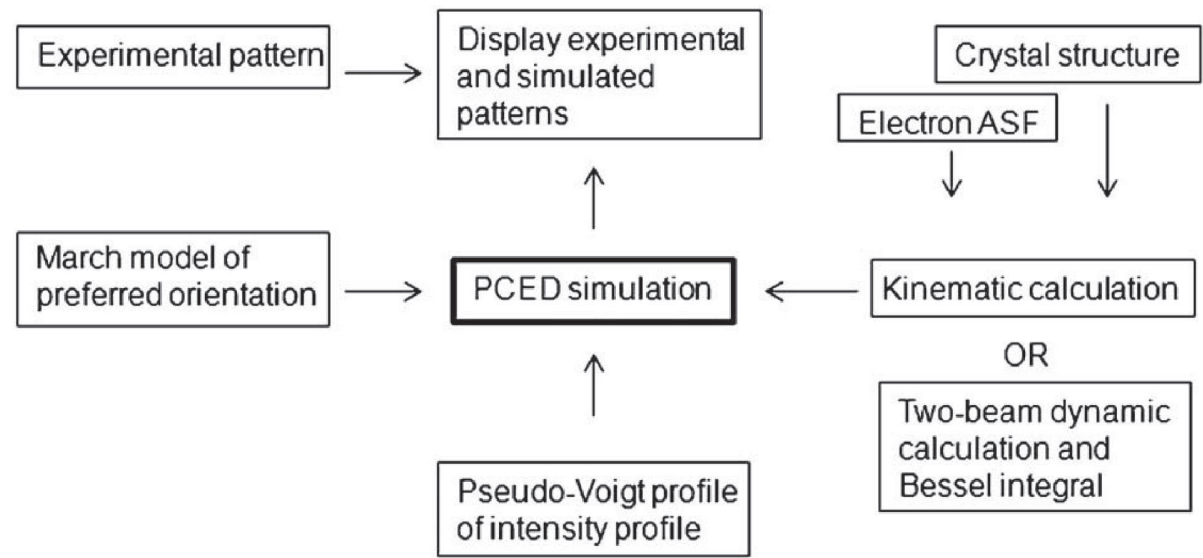

Figure 1. Flow chart of the main steps for polycrystalline electron diffraction simulation in the PCED2.0 program. 
width at half maximum (FWHM) [6] as

$$
\begin{aligned}
& G(x)=h \exp \left(-4 \ln 2\left(\frac{x-x_{0}}{F W H M}\right)^{2}\right) \\
& L(x)=\frac{h}{1+4\left[\left(x-x_{0}\right) / F W H M\right]^{2}} \\
& p V(x)=\eta G(x)+(1-\eta) L(x)
\end{aligned}
$$

where $x_{0}$ is the peak position and $\eta$ is the ratio of Gaussian and Lorentzian functions.

\subsection{March model for polycrystalline texture}

Powder samples, especially axially symmetric platy or capillary specimens, composed of effectively disk- or rod-shaped crystallites, can form with texture due to the shape of crystallites. The diffracted intensity modification due to the texture can be measured with a single pole-density profile. The March model was used for correcting the intensities from the powder X-ray diffraction of specimens with texture, which is simple but effective method for both platy and rod textures. In thin film samples, polycrystalline microstructures with out-ofplane or in-plane preferred orientations often developed during film growth. The March model can also be used to describe the diffraction intensity modification due to the polycrystalline grains with this kind of preferred growth orientation.
The formulae in the March model were originally given for X-ray powder diffraction (Bragg-Brentano geometry) in the paper by Dollase [11], which is reformulated here for the diffraction geometry of polycrystalline electron diffraction. Referring to Figure 1 in the paper by Dollase in Ref. [11], the density of a preferred zone axis for polycrystalline electron diffraction can be formulated for out-of-plane texture, as

$$
P_{g}(O)=P_{G}(\alpha)=\left(r^{2} \sin ^{2} \alpha+r^{-1} \cos ^{2} \alpha\right)^{-(3 / 2)}
$$

and for in-plane texture, as

$$
P_{g}(O)=\tilde{P}_{G}(\alpha)=\frac{2}{\pi} \int_{0}^{\pi / 2} P_{G}(\alpha, \beta) d \beta
$$

If the axial pole-density profile, $P(\varphi)$, is known, $\tilde{P}_{G}(\alpha)$ can be obtained by numerical integration making use of the relation $\varphi=\cos ^{-1}(\sin \alpha \cos \beta)$ among coordinates.

Therefore, the intensity of polycrystalline electron diffraction including texture can be expressed as

$$
I_{g}^{\text {ring }}=s \sum_{i=1}^{m} P_{G}\left(\alpha_{i}\right) I_{g}^{\text {integral }}
$$

where $s$ is the scale factor, $P_{G}\left(\alpha_{i}\right)$ the density of $g(h k l)$ poles at the scattering vectors and $\alpha_{i}$ refers to the angle between $g(h$ $k l)$ and the $i$ th member of the symmetry-equivalent set of $m$ diffraction planes. The preferred orientation plane itself is

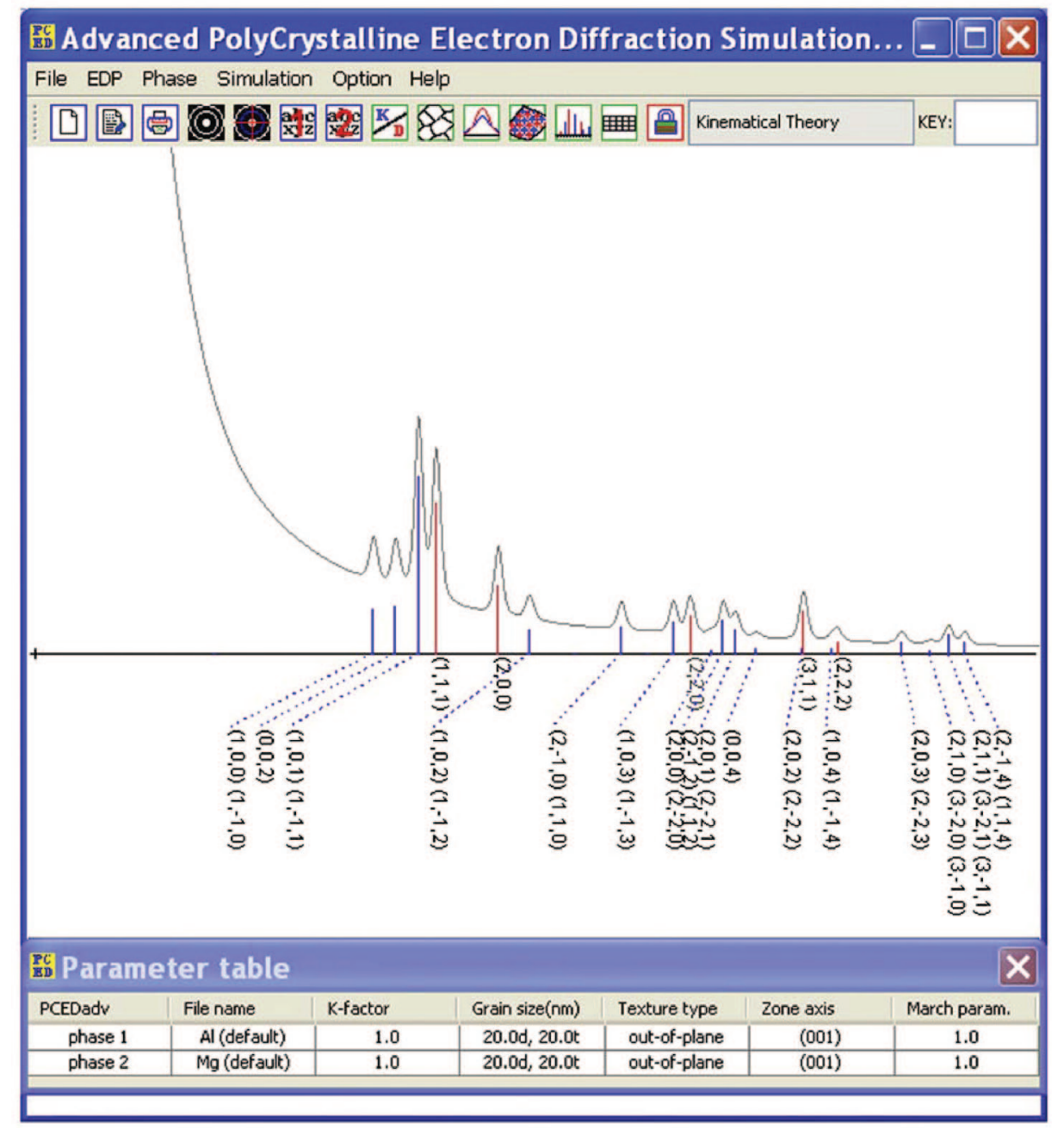

Figure 2. Screen shot of the graphical user interface of the PCED2.0. Simulated electron diffraction patterns of polycrystalline phases, fcc Al and hexagonal $\mathrm{Mg}$, are shown. 


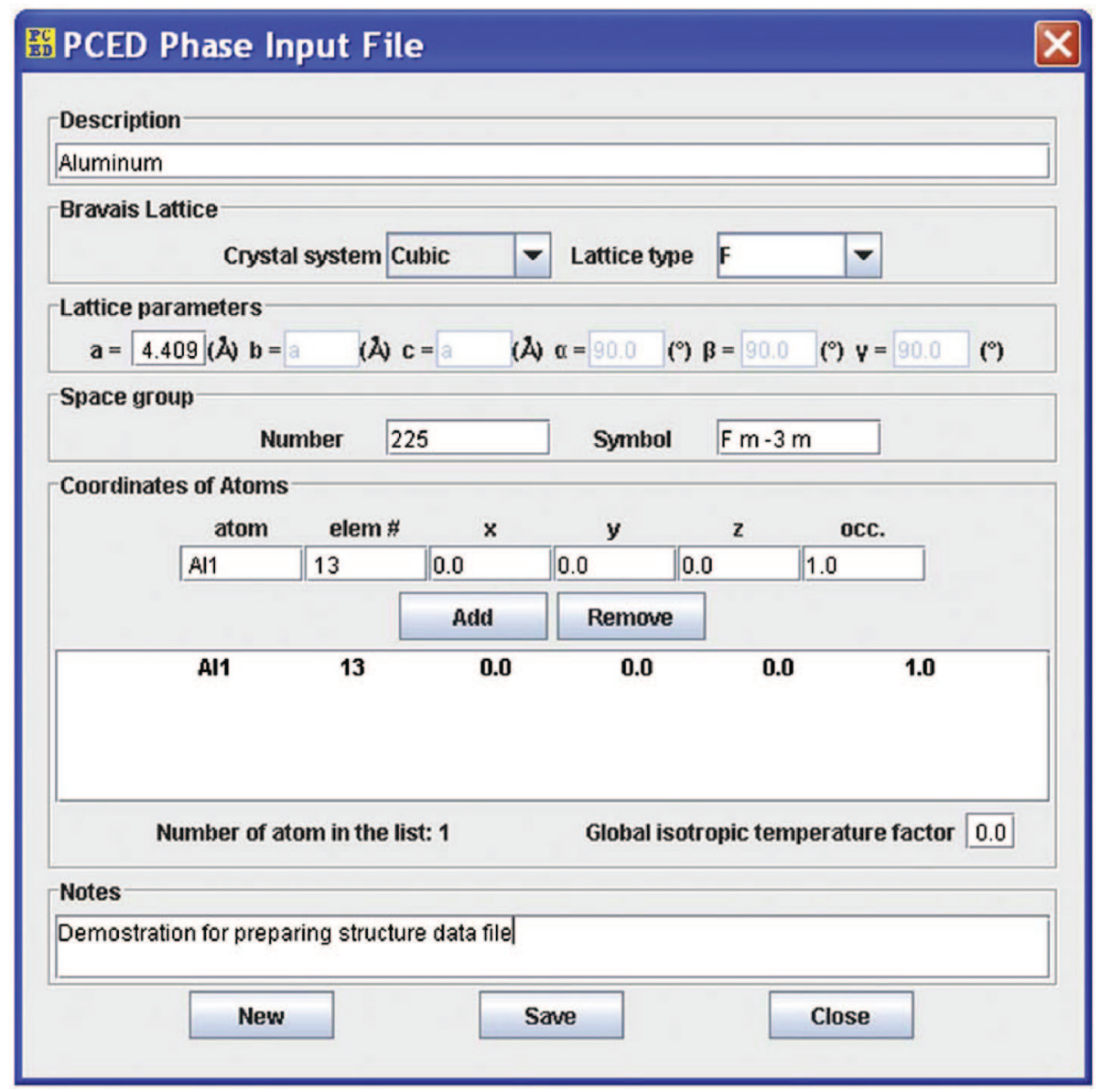

Figure 3. Screen shot of the editor tool for preparing a data file of crystalline structure in the PCED2.0.

a member of some set of symmetry-equivalent planes. If the dominant morphological feature of the crystallites is re-indexed as some other members of this set, the individual values of $\alpha_{i}$ in Equation (13) will be permuted but the sum of terms remains the same. The sum can be considered as a generalized multiplicity term.

\section{Computer program specification}

The purpose of the PCED2.0 is twofold as a teaching aid and a research tool. The program package was written in JavaTM 5 (JDK1.5), and has been tested on the Microsoft Windows $^{\mathrm{TM}}$ XP and Vista operating systems. As a Java program, the PCED2.0 is expected to run on other platforms with the Java $^{\mathrm{TM}}$ runtime environment installed.

\subsection{Program design of the PCED2.0}

The program design is described in two aspects: (i) the simulation of electron diffraction ring pattern, and (ii) the graphical user interface (GUI). The main formulae for the calculations are listed in Section 2. Numeric calculation is carried out for integrating the Bessel zeroth order function in Equation (5), and the March model for in-plane texture in Equation (12). Figure 1 shows a flow chart of the main steps in the PCED simulation. Crystal structure data and other experimental parameters are loaded to calculate diffraction intensities and ring radii with the kinematical diffraction formulae, or intensities with the Blackman dynamic formulae. The calculated intensities are saved in a data array. The simulated pattern can be generated by using the calculated intensities in combination with other conditions, such as texture models and peak pro- files. Two structure data files can be loaded at the same time either for comparison or for simulation of a two-phase system.

Figure 2 shows the GUI of the PCED2.0, which includes a drop-down menu, a toolbar, and a panel to show the results of the simulation. The menu and toolbar can be used to initiate dialog boxes for loading data or editing parameters. Most functions in the menu and toolbar are identical; however, a few functions are only provided either in the menu or in the toolbar.

A screen shot of the PCED2.0 in Figure 2 shows a simulated diffraction pattern of two phases, fcc $\mathrm{Al}$ and hexagonal $\mathrm{Mg}$. The positions of diffraction peaks are indicated by the lines; those from $\mathrm{Al}$ have their indices labeled near the abscissa and those from $\mathrm{Mg}$ are labeled away from the abscissa. The peak profile of the diffraction rings from $\mathrm{Al}$ and $\mathrm{Mg}$ phases together with an incident beam is shown above the peak lines. A table in a dialog box can be turned on to show the crystalline phases and important parameters for the simulation, as seen in the lower part of Figure 2.

Experimental diffraction patterns can be loaded into the JECP/PCED and the PCED2.0 for phase identification by directly comparing the experimental and simulated patterns. In addition, the processed 1-dimensional diffraction diagram (similar to an X-ray diffraction diagram) can also be loaded in the PCED2.0 for semi-quantitative analysis of phase fraction in a two phase system and/or texture by fitting the simulated pattern to the experimental one.

\subsection{Program features of the PCED2.0}

The usage of the PCED2.0 is elaborated in a user's manual, which is available on the author's website. Some features and basic instructions of the program are highlighted here. 


\subsubsection{Data file of a crystal structure}

Data file of a crystal structure can be prepared with a tool provided by the PCED2.0 as shown in Figure 3 or by using a text editor to modify an existed data file. It is convenient to use the editor tool since it provides assistance for users to complete the fields and also ensures compliance of the file format.

Referring Figure 3, the fields from top to bottom are described below. A text field is provided for the name of a crystalline phase. The Bravais lattice can be defined by selecting a lattice system and type from the built-in lists. Lattice types are confined to each selected crystal system. Equivalent lattice parameters are auto-filled according to the selected Bravais lattice. The range of space group numbers is restricted to be consistent with the selected Bravais lattice. Information for each atom in the crystal structure should be filled in and then added to the atom list. Modification to the list (e.g. changing parameters or deleting atom) can be easily done. The purpose for the simulation and/or a reference can be written in a note area.

A global isotropic temperature factor is used in the PCED2.0 to simulate the effect of lattice vibrations (Debye model). Although the global isotropic temperature factor is only approximate, it can be used to simulate the decrease in diffraction intensity with the magnitude of reciprocal lattice vector, $|\mathrm{g}|$, as the higher the value of $|\mathrm{g}|$, the higher the decrease in diffraction intensity.

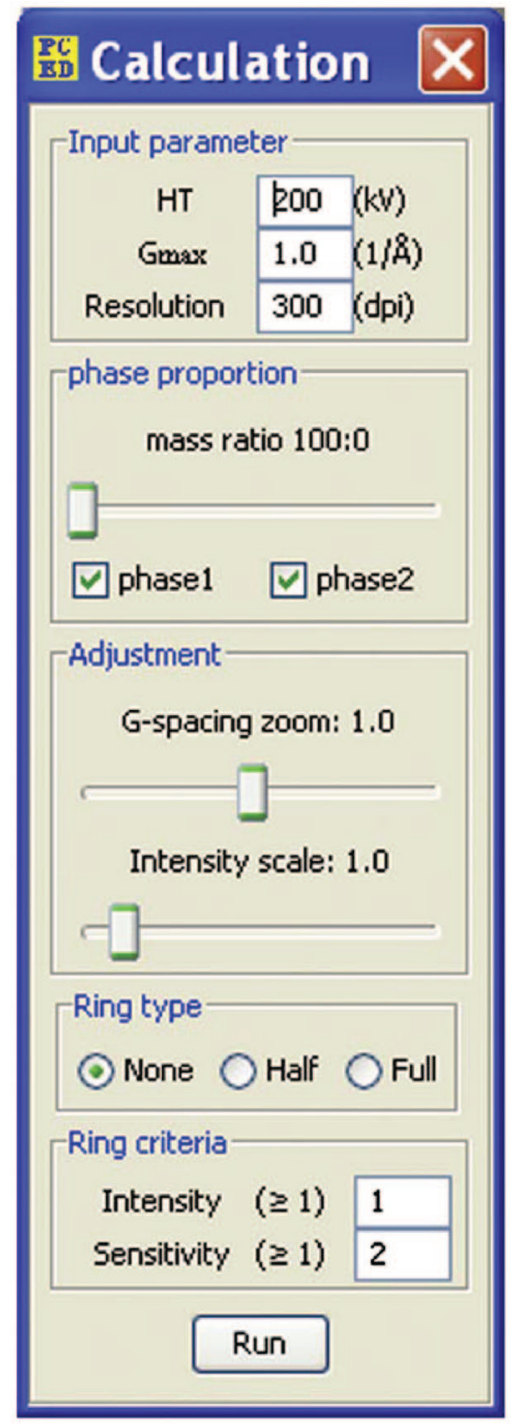

Figure 4. Screen shot of the calculation control panel in the PCED2.0.
Some atomic coordinates may not be in full occupancy in a crystal structure. In this case, the occupancy factor (default value 1.0) can be changed to a value according to the crystal structure. A partial occupancy can also be used to simulate the disordering of some atomic sites in a structure, such as the chemically ordered $\mathrm{FePt} \mathrm{L1} 1_{0}$ phase (see Section 4 ). In this case, different types of atoms may be assigned to the same atomic coordinates with different occupancy according to the chemical ordering ratio, but the sum of the occupancy factors of the two atoms is 1.0 .

\subsubsection{Simulation}

The kinematical diffraction theory is used as a default option in the simulation since it is fast and requires less input parameters. Once most of the required parameters are preliminarily set up, the Blackman correction can be utilized to do an integral two-beam dynamic calculation, which is required to further set up the average thickness of the grains and the accelerated voltage of TEM.

Basic parameters for the simulation of a diffraction pattern can be filled in or changed in the "Calculation" control panel as shown in Figure 4. The diffraction pattern is generated by the "Run" button. The pattern is updated immediately corresponding to any parameter changes, e.g., mass-ratio, scaling, texture, profile, zoom, scale, etc. Mass ratio defines mass proportion of the two phases when both phase 1 and phase 2 are selected. G-spacing zoom and intensity scale in the PCED2.0 simulate the camera length and the exposure time.

Three choices to show diffraction rings are: (i) as full ring or (ii) as half ring at the upper part in order to clearly show indices at the lower part without intervening or (iii) no ring at all. In the cases of (i) and (ii), the left side of the ring will be covered when an experimental diffraction pattern is loaded. The appearance of a diffraction ring is also controlled by the peak height itself (intensity threshold) and the peak height in comparison to neighboring points (intensity sensitivity).

There are several control panels in the PCED2.0 for inputting and changing parameters. Beside the "Calculation" control panel, the control panels for texture modeling, profile modeling, average grain-size, and flexible indexing can be accessed by using a menu or the toolbar.

As described in Section 2, the simulation of texture can be done for in-plane-textured and out-plane-textured thin films (the rod-shaped and platy for powder samples). The texture type, axis of texture, and March parameter (default value 1.0 for full random case) can be set up separately for phase1 and phase2. There are basically two situations in the study of textured samples. One is that the polycrystalline sample is expected to be randomly oriented; however, if texture exists in the experimental pattern, then the necessary correction should be carried out in the simulation for structure and phase analysis. The second is that a preferred orientation is expected with an aim to enhance the material properties, thus an extremely textured sample is under investigation and evaluation. The March parameter $(r)$ is suitable for describing the slight deviation from the random orientation in polycrystalline/powder samples; thus the higher the value, the higher the randomness. To describe the status of a preferred orientation, it is suggested to use a reverse March parameter $r^{\prime}=1.0-r$. Thus, at full randomness $r^{\prime}=0$, and the higher degree of the preferred orientation, the higher the value of $r^{\prime}$, and for perfect preferred orientation $r^{\prime}=1.0$.

The control panel for average grain-size defines two parameters for each phase: (i) the average lateral grain size, and (ii) the average grain thickness. These definitions are needed for the simulation: the average lateral grain size is associated with the FWHM of the peak while the average grain thickness is used in the Blackman formulas. These definitions also reflect in the fact that there is usually a large difference in the 


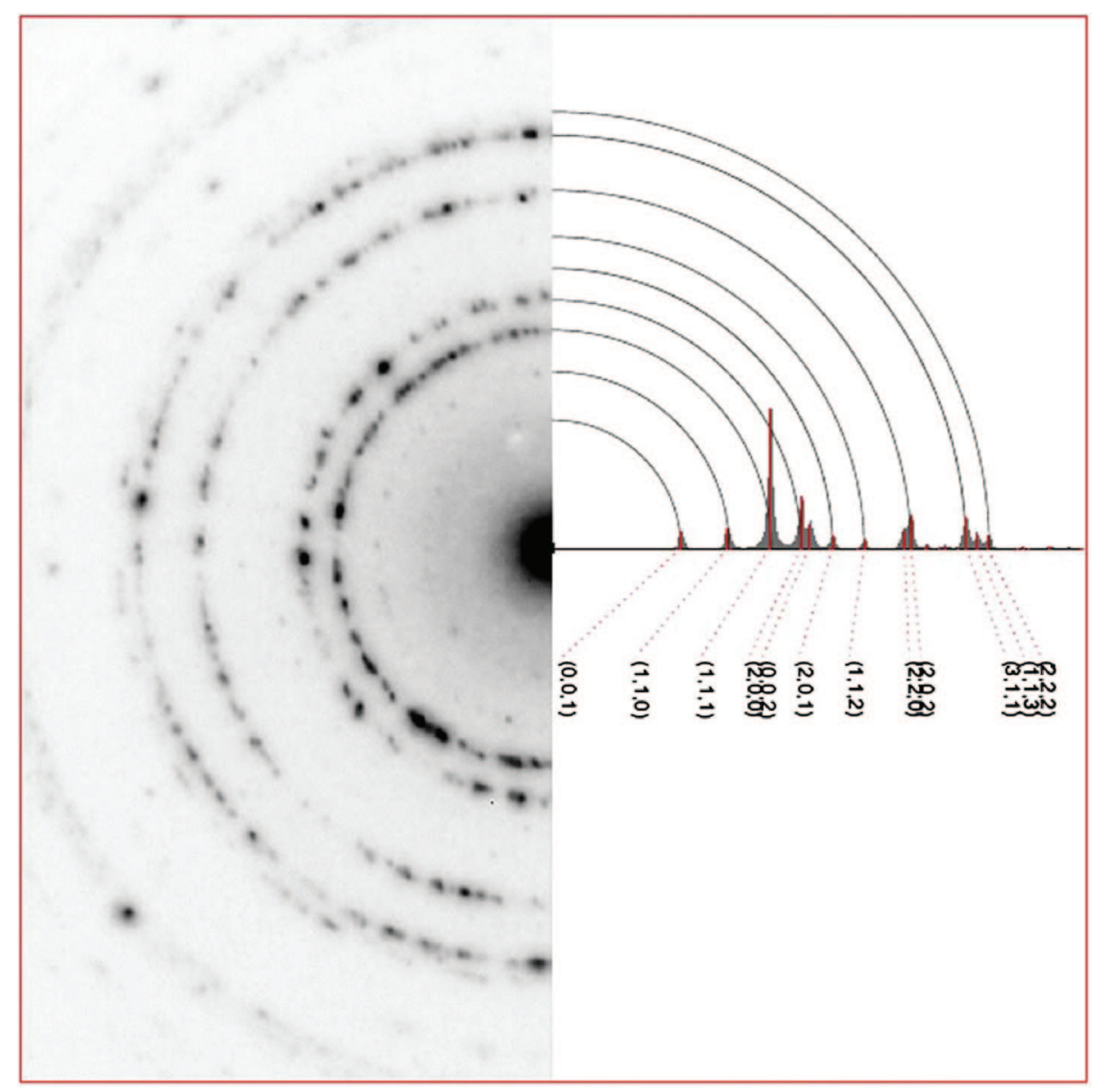

Figure 5. SAED pattern of an FePt film with a thickness of $20 \mathrm{~nm}$ of repeated Fe/Pt bilayers annealed post-deposition at $600{ }^{\circ} \mathrm{C}$ for $300 \mathrm{~s}$, together with the calculated pattern of the $\mathrm{FePt} \mathrm{L1}_{0}$ phase.

grain sizes measured in-plane and out-of-plane in thin film samples.

The peak profile of a diffraction ring is simulated using a pseudo-Voigt function in the PCED2.0. The ratio of Gaussian and Lorentzian functions in the pseudo-Voigt function can be adjusted (default value is 0.5). The FWHM is dependent on the lateral grain size $(D)$ and a constant $K, F W H M=K / D$. The peak profile can be useful for simulation of the locations of rings when there are two or more reflections close to each other in the calculated pattern. The peak position mark and a peak profile can be turned on or off separately. The style of a peak profile can be chosen as either solid (filled lines) or curve in the simulated pattern. A simulation of the incident beam can be included optionally.

In the case that the diffraction peaks are quite dense, the index labels shown in the simulated pattern may overlap if they are shown in at an area near the diffraction peaks, especially for the diffraction diagram of a two-phase system. The PCED2.0 allows users to select the location of index labels of each phase to avoid this situation.

\subsubsection{Phase identification and analysis}

For phase identification, an experimental polycrystalline electron diffraction pattern is loaded and compared with the simulated pattern, which were calculated from the presumed crystalline phase files. The experimental pattern should be in jpg (or jpeg) format and it is recommended to be square in dimensions. The experimental pattern is loaded and then centered in the display panel. A digitally processed 1-dimensional (powder X-ray diffraction diagram-like) electron diffraction diagram can be loaded for semi-quantitative structural and phase analysis.

It is simple and straight in the process to fit a simulated pattern to an experimental one since most of the parameters are independent and can be changed using the slide-bar. The simulated pattern is instantly updated corresponding to the changes in the parameters.

The PCED2.0 program can be used for the simulation of composite diffraction patterns from a two-phase system with a given weight ratio. The program converts the weight ratio to the numbers of unit-cell in diffraction with atomic weight per unit-cell for each phase. In order to reduce the effects of other experimental conditions (e.g. absorption) in the determination of the weight ratio of the two phases, it is suggested to test the result using samples with known weight ratios.

Calculated diffraction data can be saved into a text file, which includes the indices $(h k l)$, the intensities, the magnitudes of reciprocal lattice vectors $(g)$, and the crystalline plane spacings $(d)$. The simulated electron diffraction pattern together with the experimental pattern can be sent to a printer or saved into a PDF file if a PDF printer driver is installed in the computer.

\subsection{Limitation and further work}

The in-plane and out-of-plane texture can be studied experimentally by tilting samples out of a preferred zone axis to reveal more information. This technique is especially important to study the crystalline structure of textured samples since more diffraction data can be recorded in the electron diffrac- 


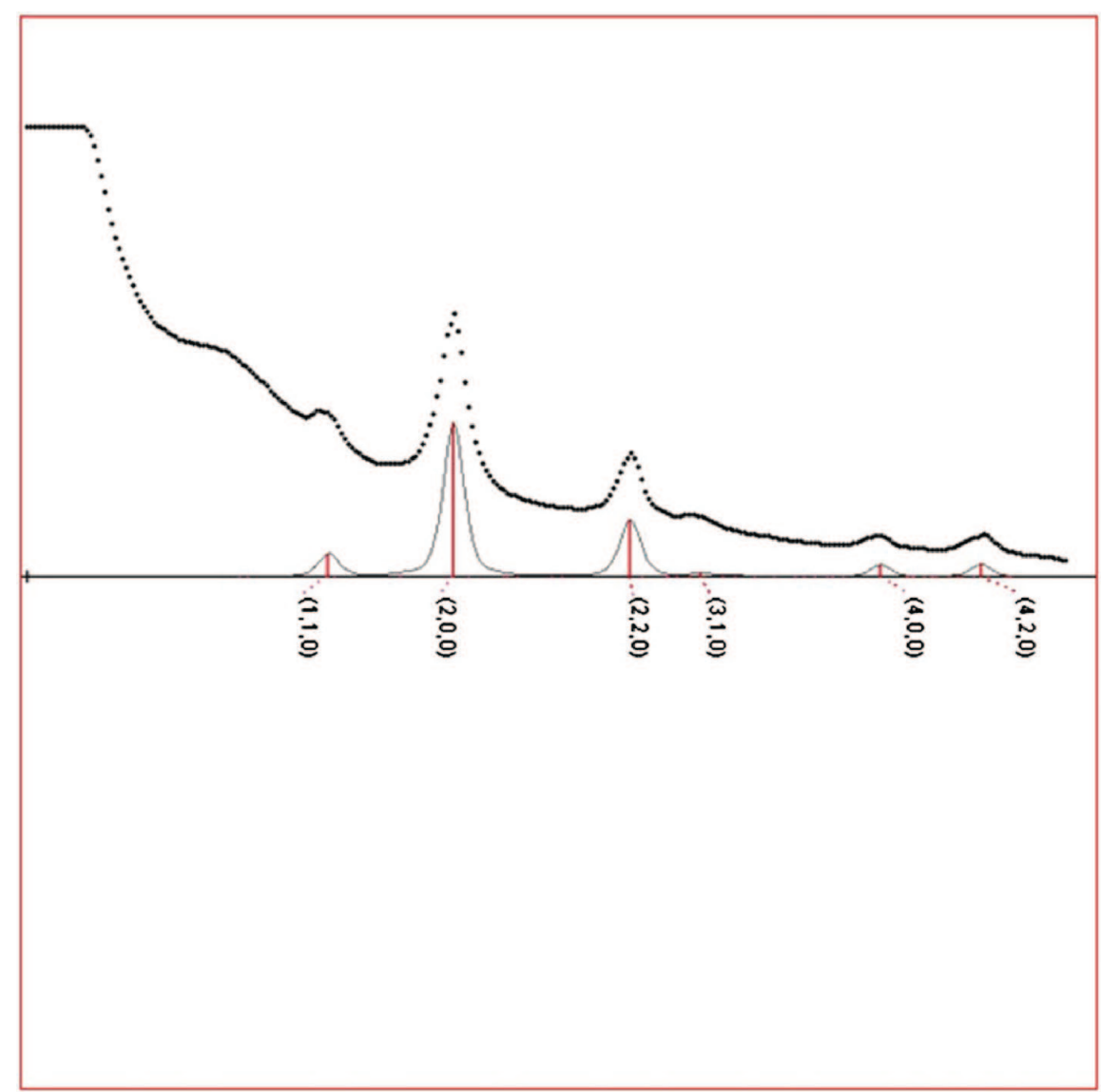

Figure 6. Diagram of digitally processed diffraction pattern of an FePt film with a thickness of $12 \mathrm{~nm}$ of repeated FePt bilayers and $1 \mathrm{~nm}$ Pt top layer annealed post-deposition at $600{ }^{\circ} \mathrm{C}$ for $300 \mathrm{~s}$, together with the calculated pattern for phase analysis.

tion patterns [19]. A program for extracting diffraction data from experimental pattern should include such a function. The PCED2.0 is designed for simulation in simple cases, thus it is limited to the simulation of texture with the incident beam parallel or vertical to the preferred zone axis.

Successful refinements of crystalline structures based on the kinematical formulae have been reported, e.g. Ref. [20, 21]. It shows that the simulations based on the kinematical formulae can be used to study ultrathin films. The Blackman formulae are integrated two-beam dynamical correction and have proven to be more accurate than the kinematical theory in dealing with polycrystalline electron diffraction data [17]. More advanced theories are expected in the near further for a more precise description of the polycrystalline electron diffraction processes. For example, it is possible to use the "Bethe potential" to include many beams as an averaging effect in the Blackman formulae. Cowley and Kuwabara [18] have shown that it is advantageous to use the multislice method to calculate electron diffraction intensities from polycrystalline materials containing heavy atoms.

Two refinement strategies exist for powder X-ray diffraction data, the Rietveld method and two-stage method [22], although the first method is more popular and widely used. Both methods can be extended for structure refinement or phase analysis of polycrystalline electron diffraction data. The recent version of ProcessDiffraction [9] adopted the Rietveld method to acquire texture and phase fraction from polycrystalline electron diffraction data. The PCED2.0 currently uses manual searching methods to fulfill a similar task. An automatic refinement routine using the two-stage method is planned for further development of the program.

\section{Examples of application}

FePt thin films composed of a chemically ordered tetragonal $\mathrm{L}_{0}$ phase are one of the most promising materials for the development of high density magnetic recording media [23]. As-deposited FePt films normally consist of the disordered fcc phase, which is a magnetically soft phase, which can be transformed into the $\mathrm{L}_{0}$ phase upon annealing. The degree of chemical order of the $\mathrm{L} 1_{0}$ phase depends on the exact chemical composition and the heat treatment conditions. For data-storage applications, it is desirable that the $\left[\begin{array}{lll}0 & 0 & 1\end{array}\right]$ axis of the $\mathrm{L1}_{0}$ phase aligns along the film normal since the [ $\left[\begin{array}{lll}0 & 1\end{array}\right]$ axis of the $\mathrm{L}_{0}$ phase is the magnetic easy axis.

The formation of the $\left(\begin{array}{lll}0 & 0 & 1\end{array}\right)$ texture for various ranges of film thicknesses was studied by selected-area electron diffraction (SAED) and analyzed with the PCED2.0. Examples are given here to demonstrate the application of this computer program.

Example 1. FePt film with a thickness of $20 \mathrm{~nm}$ : An FePt film was prepared by magnetron sputter deposition of a repeated 59 times bilayer Fe $0.16 \mathrm{~nm} / \mathrm{Pt} 0.18 \mathrm{~nm}$ and then by annealing at $600{ }^{\circ} \mathrm{C}$ for $300 \mathrm{~s}$. Figure 5 shows the SAED pattern of the FePt film with a total thickness of $20 \mathrm{~nm}$, together with the calculated pattern of the $\mathrm{FePt} \mathrm{L1} 1_{0}$ phase for phase identification. The ring radii and corresponding intensities in the calculated pattern in Figure 5 match well with the experimental pattern. On the other hand, the ring radii and corresponding intensities of the calculated pattern from the FePt disordered fcc structure (not shown here) do not match the experimental pattern. Thus, the phase is confirmed to be the chemically ordered $\mathrm{L}_{0}$ phase. The March parameter is 1.0 in the simula- 
tion, which indicates the random distribution of grains in the FePt film with thickness of $20 \mathrm{~nm}$. Fully chemically ordered FePt structure data were used in the simulation for phase identification.

Example 2. FePt film with a thickness of $12 \mathrm{~nm}$ : An FePt film was prepared by magnetron sputter deposition of a repeated 18 times bilayer Fe $0.3 \mathrm{~nm} / \mathrm{Pt} 0.3 \mathrm{~nm}$ with $1 \mathrm{~nm}$ of Pt top layer and then by annealing at $600{ }^{\circ} \mathrm{C}$ for $300 \mathrm{~s}$. Figure 6 shows the digitally processed diffraction pattern of this film with a total thickness of $12 \mathrm{~nm}$, together with the calculated pattern for phase analysis. After a few trials, a given chemical order (Pt sites with $80 \% \mathrm{Pt}+20 \% \mathrm{Fe}$; Fe sites with $80 \% \mathrm{Fe}+20 \% \mathrm{Pt}$ ) was used in the final simulation. The degree of chemical order will affect the intensities of the super-lattice reflections, e.g. ( $\left.\begin{array}{lll}0 & 0 & 1\end{array}\right)$ and $\left(\begin{array}{lll}1 & 1 & 0\end{array}\right)$, which are absent in the fully disordered structure. It is found qualitatively that the simulation matches the experimental data best when using a March parameter of 0.17 . Thus it shows that near perfect $\left(\begin{array}{lll}0 & 0 & 1\end{array}\right)$ texture of the $\mathrm{L1}_{0}$ phase has been achieved by a multilayer deposition of FePt with a $\mathrm{Pt}$ top ultrathin layer, followed by rapid thermal annealing.

\section{Conclusion}

An upgraded computer program for the simulation of polycrystalline electron diffraction patterns has been presented which can be used as a teaching aid and research tool for phase identification and semi-quantitative phase analysis. Integral two-beam dynamical (Blackman) theory, together with the March modeling for texture and pseudo-Voigt function for peak profile was adopted for an advanced simulation of the polycrystalline electron diffraction pattern. It is proved to be a fast and reliable tool for simulating polycrystalline electron diffraction patterns for phase identification and semiquantitative phase analysis by matching/fitting the experimental and simulated diffraction patterns.

Acknowledgments - The author would like to express his thanks to T. George and Professor J. Shield for their help to improve the manuscript. The collaboration with T. George and Professor D. Sellmyer on the study of the FePt thin films is one of the important driving forces for the development of the PCED2.0.

\section{References}

[1] E. Lyman and M. J. Carr, in John M. Cowley, Editor, Electron Diffraction Techniques, vol. 2, Oxford Science Publications (1993).

[2] X. Z. Li. Ultramicroscopy, 99 (2004), p. 257.

[3] J. M. Zuo. Ultramicroscopy, 66 (1996), p. 35.

[4] J. M. Zuo. Ultramicroscopy, 66 (1996), p. 21.

[5] J. L. Lábár, Proceedings of EUREM12, 2000, p. $1379 .$.

[6] X. Z. Li, Proceedings of Microanalysis and Microscopy, 2007, p. 966CD.

[7] J. L. Lábár. Microsc. Microanal., 14 (2008), p. 287.

[8] A. P. Dudka, A.S. Avilov and G.G. Lepeshov. Cryst. Rep., 53 (2008), p. 530.

[9] J. L. Lábár. Microsc. Microanal., 15 (2009), p. 20.

[10] M. Blackman. Proc. R. Soc.London, Ser. A, Math. Phys. Sci., 173 (1939), p. 68.

[11] W. A. Dollase. J. Appl. Crystallogr., 19 (1986), p. 367.

[12] J. C. H. Spence and J. M. Zuo, Electron Microdiffraction, Plenum Press (1992).

[13] L. M. Peng, G. Ren, S. L. Dudarev, and M. J. Whelan. Acta Crystallogr. A, 52 (1996), p. 257.

[14] L. M. Peng, S. L. Dudarev, and M. J. Whelan, High Energy Electron Diffraction and Microscopy, Oxford University Press (2004).

[15] K. H. Kuo, H. Q. Ye, and Y. K. Wu, Electron Diffraction Pattern: An Application in Crystallography, Science Press (1983), p. 143 (in Chinese).

[16] L. Reimer, H. Kohl, Transmission Electron Microscopy - Physics of Image Formation, Springer Series in Optical Sciences, vol. 36, 5th ed., 2008, p. 342.

[17] K. Vainshtein, B. B. Zvyagain, and A. S. Avilov, in J. M. Cowley, Editor, Electron Diffraction Techniques, vol. 1, Oxford Science Publications (1992).

[18] J. M. Cowley and S. Kuwabara. Acta Crystallogr., 15 (1962), p. 260.

[19] P. Oleynikov, S. Hovmöller, X. D. Zou, A. P. Zhukhlistov, M. S. Nickolsky, and B. B. Zvyagin. Z. Kristallogr., 219 (2004), p. 12.

[20] Th. E. Weirich, M. Winterer, S. Seifried, H. Hahn, and H. Fuess. Ultramicroscopy, 81 (2000), p. 263.

[21] Th. E. Weirich, M. Winterer, S. Seifried, and J. Mayer. Acta Crystallogr., A58 (2002), p. 308.

[22] G. Will, Powder Diffraction: The Rietveld Method and the Two-Stage Method, Springer (2006).

[23] D. Weller, A. Moser, L. Folks, M.E. Best, W. E. Lee, M. F. Toney, M. Schwickert, J.-U. Thiele, and M. F. Doerner. IEEE Trans. Magn., 36 (2000), p. 10. 